\title{
Knockdown of ATG4A inhibits breast cancer progression and promotes tamoxifen chemosensitivity by suppressing autophagy
}

\author{
QINGFANG LI ${ }^{1}$ and LINGLING ZAN ${ }^{2}$ \\ ${ }^{1}$ Division II, Department of Oncology, Yantaishan Hospital, Yantai, Shandong 264001; \\ ${ }^{2}$ Department 1 of Mammary Gland, Linyi Cancer Hospital, Linyi, Shandong 276000, P.R. China
}

Received June 23, 2021; Accepted November 17, 2021

DOI: $10.3892 / \mathrm{mmr} .2022 .12617$

\begin{abstract}
Autophagy-related 4A (ATG4A) is an autophagy regulator. The current study investigated the role of ATG4A in the development of tamoxifen-resistant breast cancer. ATG4A expression was assessed in tumor and adjacent normal tissue obtained from The Cancer Genome Atlas database. Analyses of the disease-free survival between the ATG4A high and low expression groups was then evaluated in patients with breast cancer. Cell viability and apoptosis in MCF7/R cells was detected using Cell Counting Kit-8 assay and flow cytometry, respectively. Gene set enrichment analysis identified the pathway responsible for the effects of ATG4A. The protein expression of ATG4A, LC3, p62, Bcl-2, Bax, GSK-3 $\beta$, phosphorylated (p)-GSK-3 $\beta, \beta$-catenin, cyclinD1 and c-myc in MCF and MCF7/R cells was determined using western blot. In this study, ATG4A expression was increased in the tumor tissues, and a higher ATG4A expression exhibited poor disease-free survival. While 4-hydroxytamoxifen (4-OHT) increased ATG4A expression in MCF7 and MCF7/R cells, ATG4A expression decreased in the cells treated with 3-methyladenine (3MA). Treatment with 4-OHT and rapamycin (an autophagy activator) increased the LC3-II/LC3-I ratio, LC3 puncta number and decreased the level of p62 in MCF7/R cells. However, the effects of 4-OHT and rapamycin were reversed by 3MA and knockdown of ATG4A, respectively. After treatment with 4-OHT, knockdown of ATG4A suppressed proliferation, triggered apoptosis, decreased the expression of Bcl-2, $\beta$-catenin, cyclin D1 and c-myc, and increased the expression of Bax and p-GSK3 $\beta$ in MCF7/R cells. Moreover, SKL2001, an activator of the Wnt/ $\beta$-catenin signaling pathway, reversed the effects of ATG4A knockdown on cell viability and apoptosis in MCF7/R cells. In conclusion, the knockdown of ATG4A inhibited the anticancer effects of 4-OHT in breast cancer.
\end{abstract}

Correspondence to: Dr Lingling Zan, Department 1 of Mammary Gland, Linyi Cancer Hospital, 6 Martyr Cemetery East Street, Lanshan, Linyi, Shandong 276000, P.R. China

E-mail: lingling8060@163.com

Key words: breast cancer, autophagy-related 4A, autophagy, tamoxifen

\section{Introduction}

Breast cancer is the leading cause of cancer-associated mortality (mortality rate, $11.6 \%$ ) in women worldwide (1). A total of $\sim 80 \%$ of breast tumors are estrogen receptor (ER) positive and often respond to chemotherapeutic agents, such as tamoxifen, which is a selective ER modulator (2). Tamoxifen induces cell cycle arrest and inhibits tumor growth $(3,4)$; therefore, tamoxifen therapy reduces breast cancer recurrence (5). However, tamoxifen resistance limits its clinical applications (6) and the potential mechanisms underlying tamoxifen resistance remain unclear.

Autophagy can cause drug resistance, and thus lead to a reduction in the benefits of chemotherapy (7). Alterations in autophagy have been considered to be a mechanism of tamoxifen resistance (8). The synthetic ER antagonist 4-hydroxytamoxifen (4-OHT) induces autophagy and decreases apoptosis in ER-positive breast cancer cells (9).

To date, $>30$ autophagy-related genes have been identified (10). Among them, autophagy-related (ATG) protein 4 (ATG4), a cysteine protease, serves an important role in the ATG8/LC3 lipid conjugation system, which is essential for the late step of autophagosome formation (11). The human genome contains four ATG4 homologous genes: ATG4A, ATG4B, ATG4C and ATG4D (12). Previous studies have reported that ATG4A cleaves gamma-aminobutyric acid receptor-associated protein subfamily isoforms (a subfamily of the ATG8 proteins) $(11,13)$. ATG4A is overexpressed in multiple human cancers, such as osteosarcoma (14) and gastric cancer (15), and its overexpression is often associated with the progression of malignancy. A previous study has reported that ATG4A participates in stem cell maintenance during breast cancer (16). Therefore, an improved understanding of the role of ATG4A during tamoxifen resistance would be beneficial in treatment planning for patients undergoing chemotherapy to treat breast cancer.

In the present study, it was aimed to investigate whether interference with ATG4A could affect autophagy and 4-OHT sensitivity in breast cancer cell lines and elucidate its underlying molecular mechanisms.

\section{Materials and methods}

Cell culture and treatment. Human breast cancer MCF7 cells (The Cell Bank of Type Culture Collection of The Chinese Academy of Sciences) were cultured in DMEM: 
F-12 (HyClone; Cytiva) containing 10\% FBS (HyClone; Cytiva), $100 \mathrm{U} / \mathrm{ml}$ penicillin (Thermo Fisher Scientific, Inc.), $100 \mathrm{mg} / \mathrm{ml}$ streptomycin (Thermo Fisher Scientific, Inc.), $2 \mathrm{mM}$ GlutaMax (HyClone; Cytiva) and $6 \mathrm{ng} / \mathrm{ml}$ insulin (HyClone; Cytiva) at $37^{\circ} \mathrm{C}$ and $5 \% \mathrm{CO}_{2}$. MCF7/TAMR-7 cells (MCF7/R; Ximbio), which are tamoxifen-resistant, were cultured in the presence of $1 \mu \mathrm{M} 4-\mathrm{OHT}$ (Sigma-Aldrich; Merck $\mathrm{KGaA}$ ) under the same conditions as aforementioned.

MCF7 and MCF7/R cells were divided into control, 4-OHT and 4-OHT+3-methyladenine (3MA) groups. Cells in the 4-OHT group were treated with $10 \mu \mathrm{M} 4-\mathrm{OHT}$ with DMSO as the vehicle. Cells in 4-OHT+3-MA group were treated with $10 \mu \mathrm{M} 4-\mathrm{OHT}$ and $1 \mathrm{mM}$ 3MA (Sigma-Aldrich; Merck KGaA) with DMSO as the vehicle. MCF7/R cells in rapamycin group were treated with $10 \mathrm{nM}$ rapamycin (Selleck Chemicals) with DMSO as the vehicle. Cells in control group were treated with DMSO in follow up experiments.

Lentiviral infection and treatment. Lentiviral plasmid (GV248; GeneChem, Inc.) carrying the knockdown vector of ATG4A [short hairpin (sh)-ATG4A] and empty vector were purchased from Shanghai GenePharma Co., Ltd. The sequences of sh-ATG4A and sh-negative control (NC) were as follows: sh-ATG4A sense, 5'-GGATGTTTGAATTAG TTCA-3' and antisense, 5'-TGAACTAATTCAAACATCC-3'; and sh-NC sense, 5'-TTCTCCGAACGTGTCACGTTT-3' and antisense, 5'-ACGTGACACGTTCGGAGA ATT-3'. A second-generation system was used in this experiment. Briefly, 293T cells (The Cell Bank of Type Culture Collection of The Chinese Academy of Sciences) were seeded into six-well plates $\left(4 \times 10^{5}\right.$ cells/well) and transfected with lentiviral plasmid, psPAX2 (Biovector NTCC Inc.) and PMD2G (Biovector NTCC Inc.) in the following ratio: 10:7.5:2.5 $\mu \mathrm{g}$. Transfection was conducted using lipofectamine ${ }^{\circledR} 2000$ (Invitrogen; Thermo Fisher Scientific, Inc.) at $37^{\circ} \mathrm{C}$ for $48 \mathrm{~h}$. Next, lentiviral particles were collected after centrifugation at $50,000 \times \mathrm{g}$ for $2 \mathrm{~h}$. MCF7/R cells $\left(5 \times 10^{4}\right.$ cells/well) were seeded into 24-well plates and then infected with lentivirus supernatant $\left(1 \times 10^{8} \mathrm{TU} / \mathrm{ml}\right)$ at a multiplicity of infection of 20 in the presence of $8 \mathrm{mg} / \mathrm{ml}$ polybrene (MilliporeSigma). After $48 \mathrm{~h}$ of incubation, stably transfected cell lines were selected with $2.5 \mu \mathrm{g} / \mathrm{ml}$ puromycin (Sigma-Aldrich; Merck KGaA) for 1 week. The stably transfected cell lines were maintained under $0.5 \mu \mathrm{g} / \mathrm{ml}$ puromycin. After transfection for $24 \mathrm{~h}$, subsequent experiments were performed. Cells were treated with 4-OHT (10 $\mu \mathrm{M})$. Additionally, $20 \mu \mathrm{M}$ SKL2001 (MedChemExpress) was added to the 4-OHT + sh-ATG4A group.

Cell Counting Kit-8 (CCK-8) assay. MCF7/R cells $\left(2 \times 10^{3}\right.$ cells/well) were incubated at $37^{\circ} \mathrm{C}$ and $5 \% \mathrm{CO}_{2}$ for $24 \mathrm{~h}$ after exposure to tamoxifen $(10 \mu \mathrm{M})$. The cells were then cultured with $10 \mu \mathrm{l} \mathrm{CCK}-8$ solution (Beyotime Institute of Biotechnology) for $2 \mathrm{~h}$ at $37^{\circ} \mathrm{C}$. The absorbance was measured at $450 \mathrm{~nm}$ using a microplate reader (Dynex Technologies, Inc.).

Flow cytometric analysis of apoptosis. According to the manufacturer's instructions, cell apoptosis was detected using an Annexin V-FITC kit (Beyotime Institute of Biotechnology). Briefly, MCF-7/R cells ( $5 \times 10^{4}$ cells/well) were harvested and centrifuged at $1,000 \mathrm{x} \mathrm{g}$ for $5 \mathrm{~min}$ at room temperature. Cells were then stained with Annexin V-FITC and propidium iodide for $15 \mathrm{~min}$ at room temperature in the dark. Finally, apoptosis was analyzed using a BD Accuri ${ }^{\mathrm{TM}}$ C6 Plus flow cytometer (BD Biosciences) and the rate of apoptosis was calculated using BD Accuri C6 Software (BD Biosciences).

Green fluorescent protein (GFP)-LC3 analysis. MCF7 and $\mathrm{MCF} 7 / \mathrm{R}$ cells were seeded into 12 -well plates $\left(6 \times 10^{4}\right.$ cells/well) at $37^{\circ} \mathrm{C}$ overnight, and then transfected with the GFP-LC3 expression plasmids ( $2 \mu \mathrm{g} / \mathrm{l}$; LC3; Hanbio Biotechnology Co., Ltd.) using lipofectamine ${ }^{\circledR} 2000$ at $37^{\circ} \mathrm{C}$ for $24 \mathrm{~h}$. After transfection for $24 \mathrm{~h}$, cells were treated with $4-\mathrm{OHT}(10 \mu \mathrm{M}), 3 \mathrm{MA}$ $(1 \mathrm{mM})$ or rapamycin $(10 \mathrm{nM})$ for $24 \mathrm{~h}$. Cells were then fixed with $4 \%$ formaldehyde for $10 \mathrm{~min}$ at room temperature and observed under a confocal microscope (Leica Microsystems $\mathrm{GmbH})$. The number of LC3 puncta was counted using at least 20 cells from six randomly selected fields, and each experiment was repeated three times.

Western blot analysis. MCF7 and MCF7/R cells were lysed in a RIPA lysis buffer (Sigma-Aldrich; Merck KGaA). According to the manufacturer's protocol, NE-PER nuclear and cytoplasmic extraction reagents (Thermo Scientific Pierce) was used to extract nuclear and cytoplasmic protein from MCF7/R cells. Equal amounts of protein samples $(30 \mu \mathrm{g}$ per lane) were separated via $12 \%$ SDS-PAGE and transferred to PVDF membranes (EMD Millipore). After blocking using TBS with $0.05 \%$ Tween-20 (TBST) containing 5\% non-fat milk at room temperature for $2 \mathrm{~h}$, the membranes were incubated with the following primary antibodies: ATG4A (cat. no. ab108322; 1:1,000; Abcam), LC3 (cat. no. 3868, $1: 1,000)$, p62 (cat. no. 8025; 1:1,000), phosphorylated (p)-GSK-3 $\beta$ (cat. no. 9322; 1:1,000; all from Cell Signaling Technology, Inc.), GSK-3 $\beta$ (cat. no. ab32391; 1:1,000; Abcam), $\beta$-catenin (cat. no. 8480; 1:1,000; Cell Signaling Technology, Inc.), cyclin D1 (cat. no. 55506, 1:1,000; Cell Signaling Technology, Inc.), c-myc (cat. no. ab32072; 1:1,000; Abcam), Bax (cat. no. 5023; 1:1,000), Bcl-2 (cat. no. 3498; 1:1,000), GAPDH (cat. no. 5174; 1:1,000), histone H3 (cat. no. 4499; 1:1,000; all from Cell Signaling Technology, Inc.) and $\beta$-actin (cat. no. ab179467; 1:1,000; Abcam) at $4^{\circ} \mathrm{C}$ overnight. After washing with TBST, the membranes were incubated with horseradish peroxidase-labelled secondary antibody (cat. no. 7074; 1:2,000; Cell Signaling Technology, Inc.) at room temperature for $1 \mathrm{~h}$. The bands were visualized using Pierce ${ }^{\mathrm{TM}}$ ECL Plus Western Blotting Substrate (Pierce; Thermo Fisher Scientific, Inc.) and detected with ImageJ v1.8.0 (National Institutes of Health).

Immunofluorescence. MCF7/R cells were fixed with $4 \%$ paraformaldehyde for $20 \mathrm{~min}$ at room temperature and incubated with $0.3 \%$ Triton $\mathrm{X}-100$ for $30 \mathrm{~min}$ at room temperature. The cells were incubated with primary antibodies targeting $\beta$-catenin (1:200; cat. no. 8480; Cell Signaling Technology, Inc.) overnight at $4^{\circ} \mathrm{C}$ and then incubated with goad anti-rabbit Alexa Fluor 488 (1:300; cat. no. A11008; Thermo Fisher Scientific, Inc.) for $1 \mathrm{~h}$ at $37^{\circ} \mathrm{C}$. The cells were then incubated with $5 \mu \mathrm{g} / \mathrm{ml}$ DAPI for $20 \mathrm{~min}$ at room temperature and observed under a fluorescence microscope (Leica Microsystems $\mathrm{GmbH}$ ). 
The Cancer Genome Atlas (TCGA) database analysis. ATG4A expression data were obtained from the TCGA database (https://tcga-data.nci.nih.gov/tcga/) using the R package TCGAbiolinks (17). These data included 1,097 breast cancer samples and 114 adjacent normal samples. Subsequently, the clinical information of 439 patients with breast cancer were downloaded from the TCGA database. After excluding 8 patients with breast cancer with incomplete relevant information, 431 patients were divided into two groups based on the median value of ATG4A levels. The clinical characteristics (age and TNM stage) of the patients were analyzed. After excluding 73 patients with breast cancer with an overall survival $\leq 30$ days, 358 patients with breast cancer were divided into two groups (high and low) based on a group cut-off of 'quartile'. Survival analysis was performed with the R package 'survival' (18) using the Kaplan-Meier method.

Gene set enrichment analysis (GSEA). To further explore the pathway in which ATG4A may be involved, the Molecular Signatures Database (v7.4; https://www.gsea-msigdb. org/gsea/msigdb/index.jsp) hallmark gene set was used to perform GSEA. GSEA parameters were set as follows: Number of permutations $=1,000$; permutation type $=$ gene set; enrichment statistic=weighted; metric for ranking genes=Signal2Noise. Gene sets with a positive (or negative) enrichment score and $\mathrm{P}<0.05$ after 1,000 permutations were considered as significantly enriched gene sets.

Statistical analysis. Data are presented as the mean \pm standard deviation of three experiments. Computer-based calculations were conducted using GraphPad Prism 7 (GraphPad Software, Inc.). Statistical analysis was performed using unpaired Student's t-test and one-way analysis of variance with Tukey's post hoc test. $\chi^{2}$ test was used to assess the relationship between ATG4A expression and clinicopathological features in patients with breast cancer. Kaplan-Meier analysis was performed using log-rank test. $\mathrm{P}<0.05$ was considered to indicate a statistically significant difference.

\section{Results}

ATG4A levels are associated with the survival of patients with breast cancer in The Cancer Genome Atlas (TCGA) database. To investigate the clinical relevance of ATG4A expression, ATG4A expression in TCGA database was firstly analyzed. The results revealed that the expression of ATG4A was higher in breast cancer tissues than in a number of adjacent normal tissues (Fig. 1A). Subsequently, the clinical characteristics (age and TNM stage) of the patients were analyzed. As demonstrated in Table I, high expression of ATG4A was significantly associated with age, but not with TNM stage. In addition, the results of Kaplan-Meier survival analysis revealed that patients with a higher ATG4A expression exhibited poor disease-free survival (Fig. 1B). These results indicated that ATG4A may be involved in the pathogenesis of breast cancer.

ATG4A expression and autophagy are enhanced in MCF7/R cells. To explore the molecular mechanism of 4-OHT resistance in breast cancer cells, a 4-OHT-resistant breast cancer cell line (MCF7/R) was used. It was investigated whether
Table I. Relationship between ATG4A expression and clinicopathological features in patients with breast cancer.

\begin{tabular}{lccc}
\hline & \multicolumn{2}{c}{ ATG4A } & \\
\cline { 2 - 3 } Characteristic & $\begin{array}{c}\text { Low } \\
\text { expression, } n\end{array}$ & $\begin{array}{c}\text { High } \\
\text { expression, } n\end{array}$ & P-value \\
\hline Age, years & & & 0.0006 \\
$<50$ & 89 & 55 & \\
$\geq 50$ & 127 & 160 & 0.5786 \\
TNM stage & & & \\
I/II & 166 & 170 & \\
III/IV & 50 & 45 & \\
\hline
\end{tabular}

ATG4A, autophagy-related 4A.

ATG4A mediated the regulatory effect of autophagy on 4-OHT resistance in breast cancer. MCF7 and MCF7/R cells were treated with the autophagy inhibitor $3 \mathrm{MA}$ and the expression of ATG4A and autophagy genes in vitro was detected. The results revealed that treatment with 4-OHT increased ATG4A expression in MCF7 and MCF7/R cells compared with control group, while treatment with 3MA decreased ATG4A expression compared with 4-OHT group (Fig. 2A). The LC3-II/LC3-I ratio increased and the level of p62 decreased in MCF7/R cells compared with that in MCF7 cells. Compared with control group, treatment with 4-OHT significantly increased the LC3-II/LC3-I ratio and decreased the level of p62, particularly in MCF7/R cells; however, this effect of 4-OHT was reversed by 3MA (Fig. 2B). In addition, the number of LC3 puncta increased in MCF7 and MCF7/R cells after treatment with 4-OHT, but decreased after treatment with 3MA (Fig. 2C).

ATG4A knockdown enhances the sensitivity of breast cancer cells to 4-OHT. To investigate the role of ATG4 in 4-OHT resistance in breast cancer cells, MCF7/R cells were transfected with sh-ATG4 to establish a stable knockdown ATG4A cell line. As demonstrated in Fig. 3A and B, ATG4A expression was significantly inhibited in MCF7/R cells transfected with sh-ATG4. CCK-8 and flow cytometric apoptosis analysis revealed that there was no significant difference in cell viability and apoptosis between the control and 4-OHT groups, while the knockdown of ATG4A reduced viability and apoptosis in MCF7/R cells treated with 4-OHT (Fig. 3C and D). Additionally, the expression of apoptosis-related proteins (Bcl-2 and Bax) was analyzed via western blot analysis. The results demonstrated that the knockdown of ATG4A decreased Bcl-2 expression and increased Bax expression (Fig. 3E).

ATG4A knockdown attenuates tamoxifen-induced autophagy. Previous studies have demonstrated that ATG4A is closely related to autophagy (19-21). However, the role of ATG4A during autophagy in breast cancer cells remains unclear. To further confirm the role of ATG4A in autophagy, MCF7/R cells were transfected with sh-ATG4 to perform loss-of-function analysis. As revealed in Fig. 4A and C, after treatment with 4-OHT, 

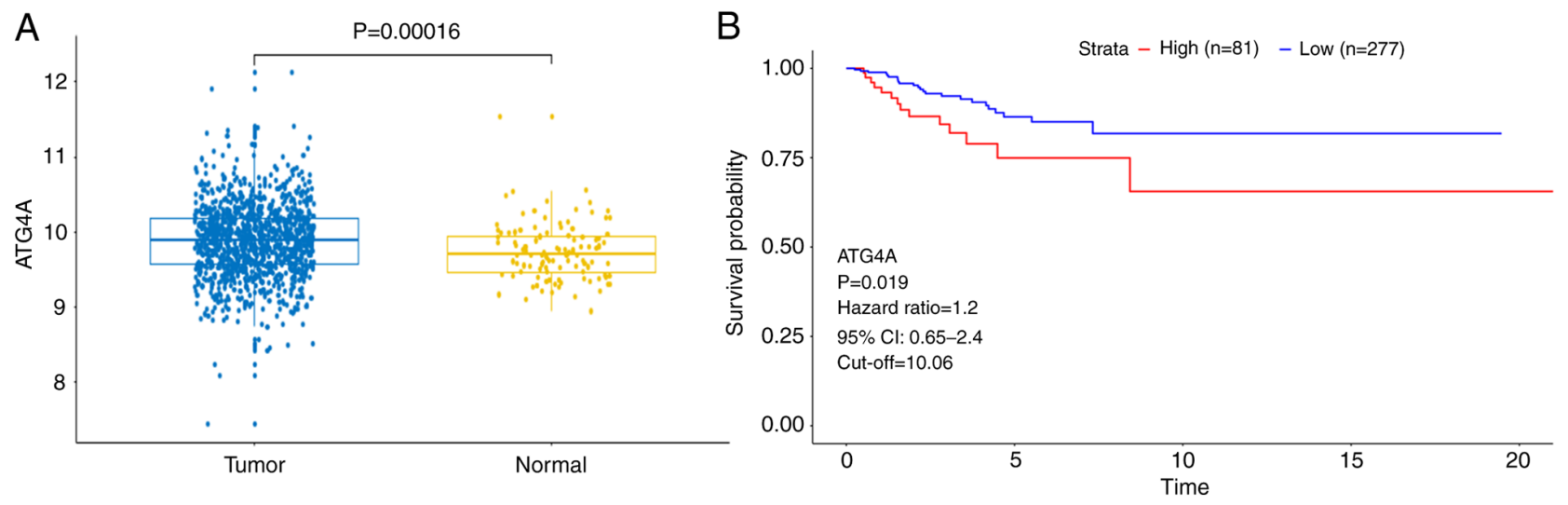

Figure 1. ATG4A is associated with the survival of patients with breast cancer in the TCGA database. (A) ATG4A expression in primary solid tumor tissue (tumor; 1,097) and adjacent normal solid tissue (normal; 114) were downloaded from TCGA database. (B) Kaplan-Meier survival analysis of disease-free survival between the ATG4A high and low expression groups in 358 patients with breast cancer from TCGA database. ATG4A, autophagy-related 4A; TCGA, The Cancer Genome Atlas.
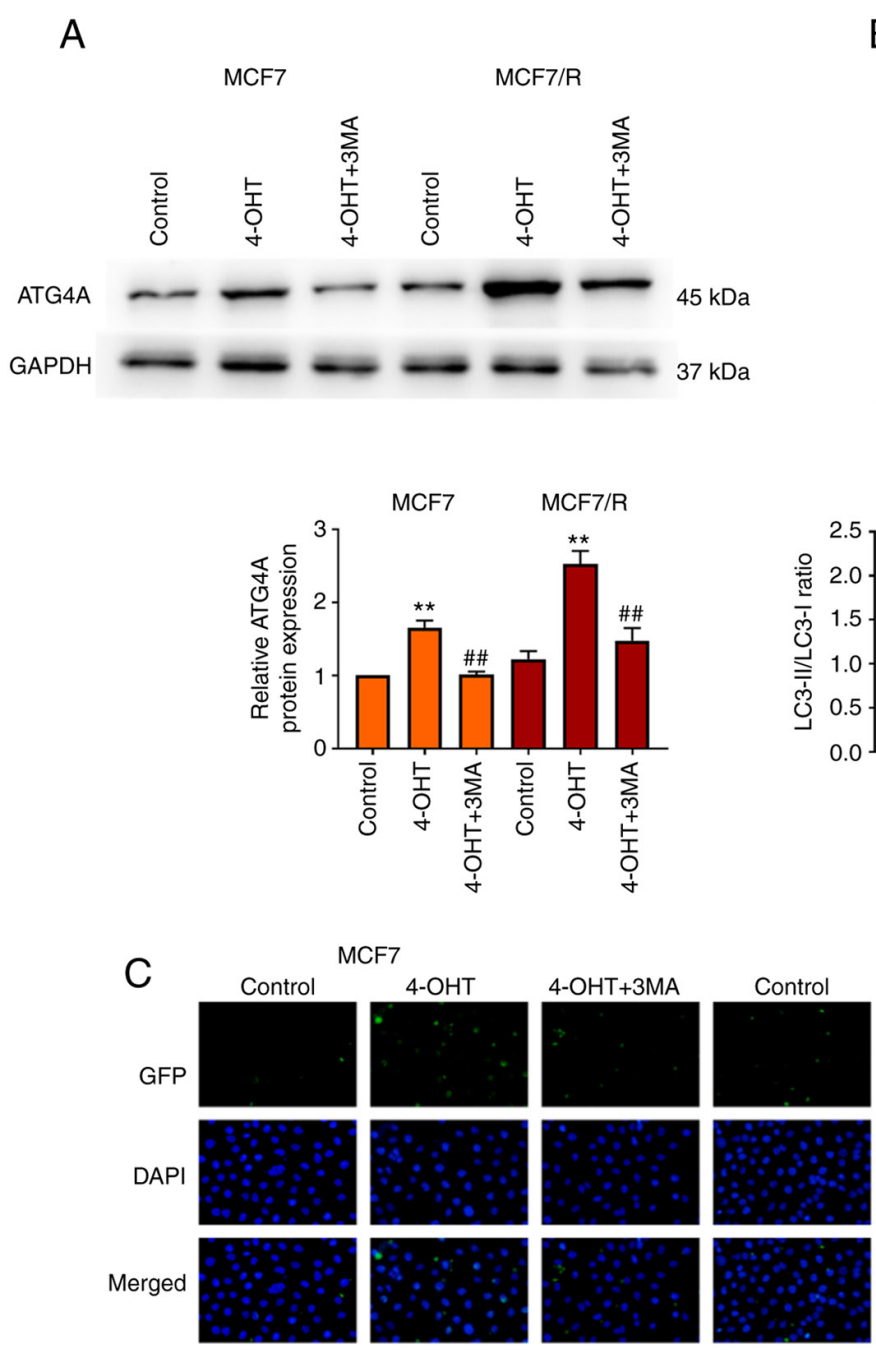

B

MCF7 MCF7/R
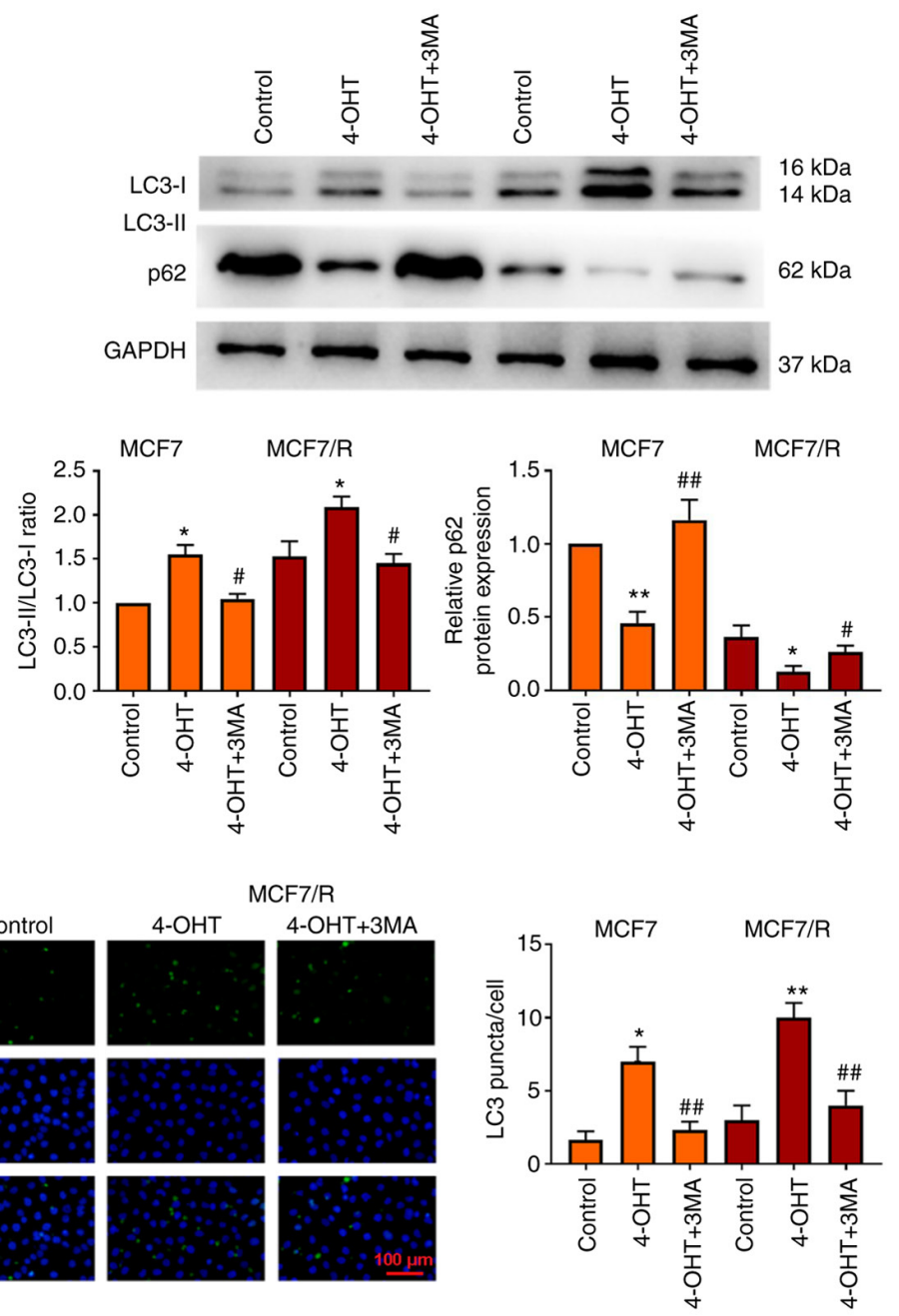

Figure 2. ATG4A expression and autophagy are enhanced in MCF7/R cells. The expression of (A) ATG4A and (B) LC3 and p62 in MCF and MCF7/R cells was determined using western blot analysis. (C) The number of LC3-puncta in MCF and MCF7/R cells was counted (scale bar, $100 \mu \mathrm{m}$ ). "P<0.05, ${ }^{* *} \mathrm{P}<0.01$ vs. control group; ${ }^{*} \mathrm{P}<0.05$ and ${ }^{\# \#} \mathrm{P}<0.01$ vs. 4-OHT group. ATG4A, autophagy-related 4A; 4-OHT, 4-hydroxytamoxifen; 3MA, 3-methyladenine; GFP, green fluorescent protein.

the knockdown of ATG4A decreased the LC3-II/LC3-I ratio, increased the level of p62, and decreased the number of LC3 puncta compared with 4-OHT + sh-NC group. After treatment with rapamycin (an autophagy activator), the LC3II/LC3I ratio 

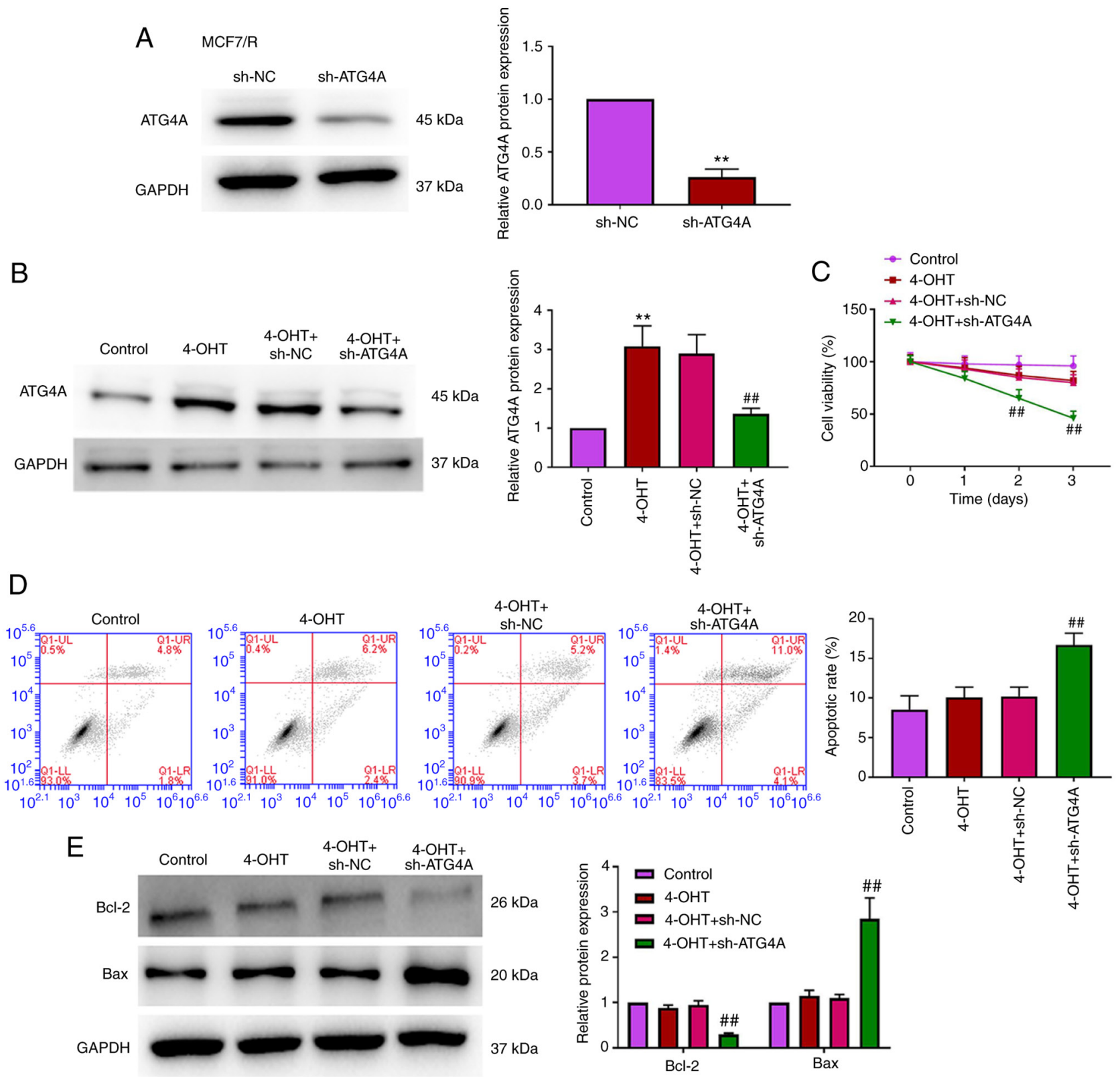

Figure 3. Knockdown of ATG4A enhances the sensitivity of breast cancer cells to tamoxifen. (A and B) The expression of ATG4A in MCF7/R cells was determined using western blot analysis. (C) Cell viability was detected with Cell Counting Kit-8 assay. (D) Apoptosis was detected using flow cytometric analysis. (E) The expression of apoptosis-related proteins (Bcl-2 and Bax) was analyzed using western blot analysis. ${ }^{* *} \mathrm{P}<0.01$ vs. control group; ${ }^{\# \#} \mathrm{P}<0.01$ vs. 4-OHT + sh-NC group. ATG4A, autophagy-related 4A; 4-OHT, 4-hydroxytamoxifen; sh-, short hairpin; NC, negative control.

increased, p62 level decreased, and the number of LC3 puncta increased compared with control group. The knockdown of ATG4A reversed the effect of rapamycin on the LC3-II/LC3-I ratio, level of p62 and number of LC3 puncta (Fig. 4B and D).

ATG4A knockdown inhibits the Wnt/ $\beta$-catenin pathway. To further explore the mechanism of ATG4A in breast cancer cells, GSEA was performed to identify the pathway responsible for the effects of ATG4A. The results indicated that ATG4A was enriched in the Wnt/ $\beta$-catenin pathway (Fig. 5A). The Wnt/ $\beta$-catenin pathway serves an important role in cancer progression and chemoresistance (22). Compared with the 4-OHT+sh-NC group, the knockdown of ATG4A increased p-GSK3 $\beta$ expression and decreased the expression of $\beta$-catenin, cyclin D1 and c-myc in MCF7/R cells (Fig. 5B).
Compared with the 4-OHT+sh-NC group, the knockdown of ATG4A decreased the expression of $\beta$-catenin in the nucleus (Fig. 5C and D). SKL2001 is an activator of the Wnt/ $\beta$-catenin signaling pathway (23). Treatment with SKL2001 reversed the effect of ATG4A knockdown on cell viability, apoptosis and the Wnt/ $\beta$-catenin signaling pathway in MCF7/R cells (Fig. 5E-G).

\section{Discussion}

Breast cancer is the most common malignant cancer in women (24). Although surgery and radiotherapy have been indicated to be curative for certain early stage cases, chemotherapy remains a common and essential therapeutic method in breast cancer $(25,26)$. Long-term clinical observations have 
A

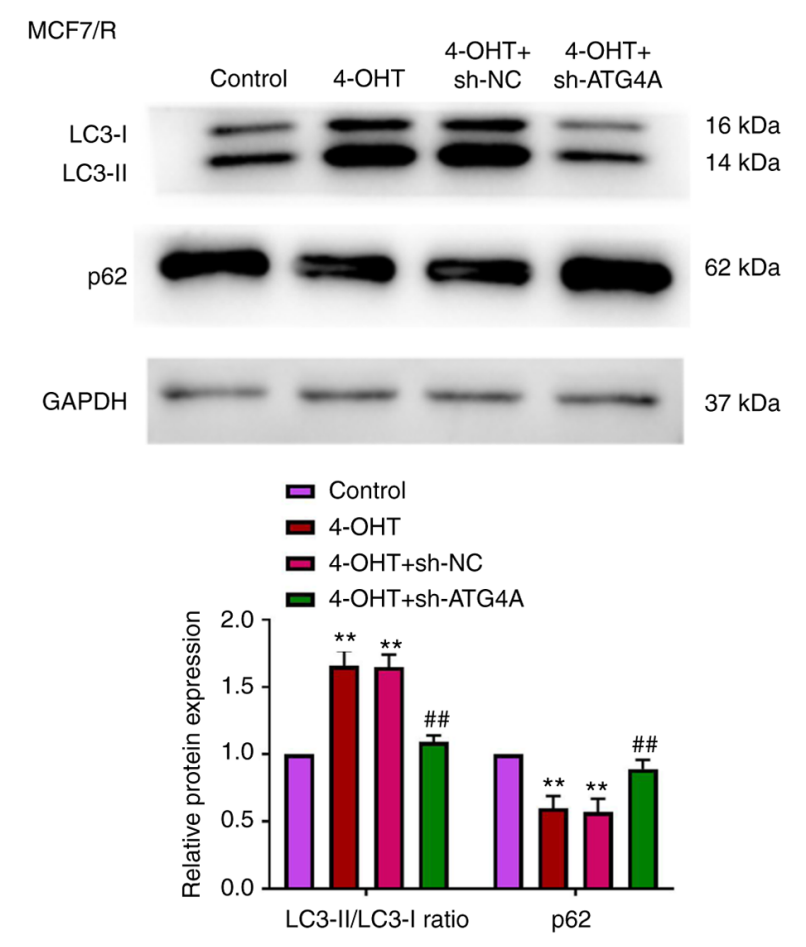

C
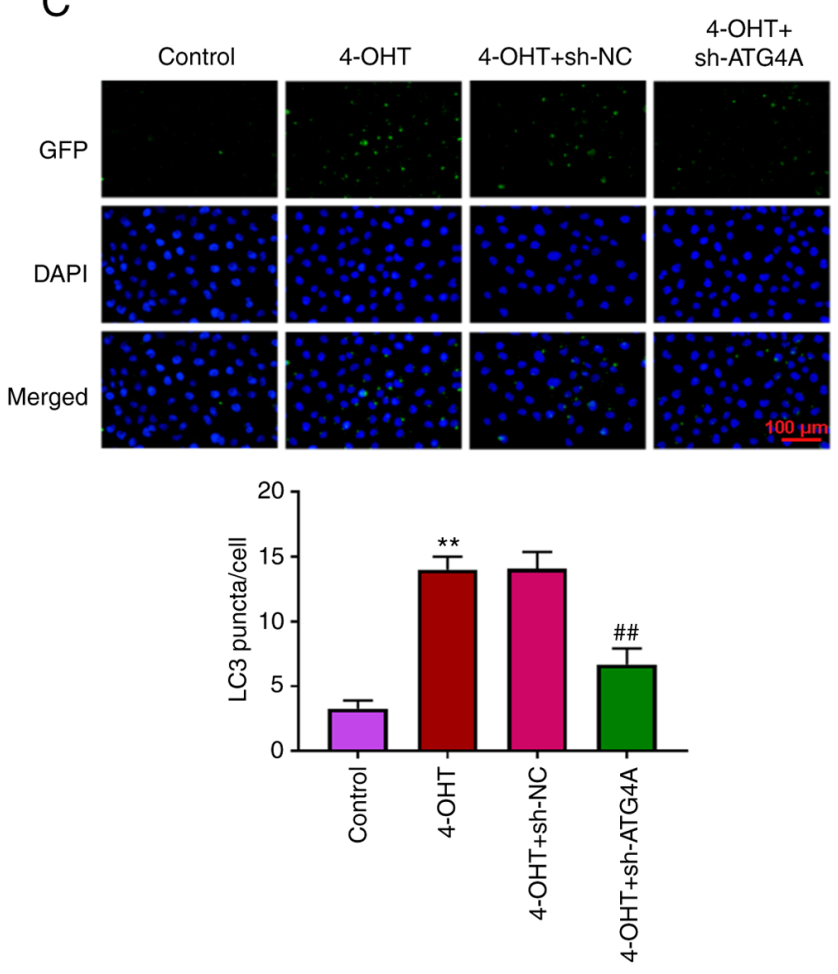

B
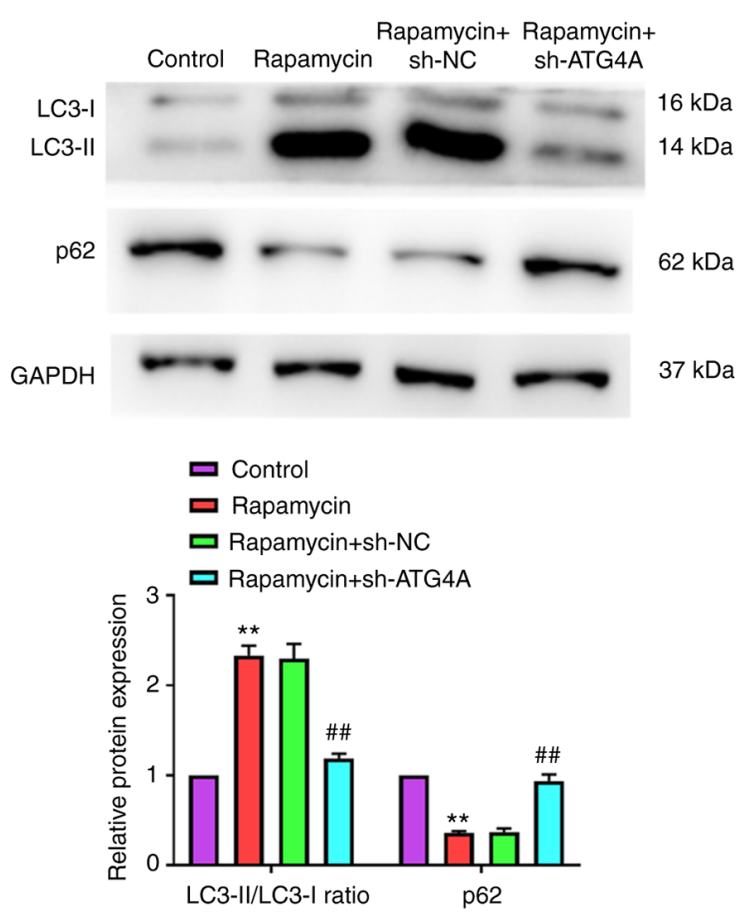

$\mathrm{D}$
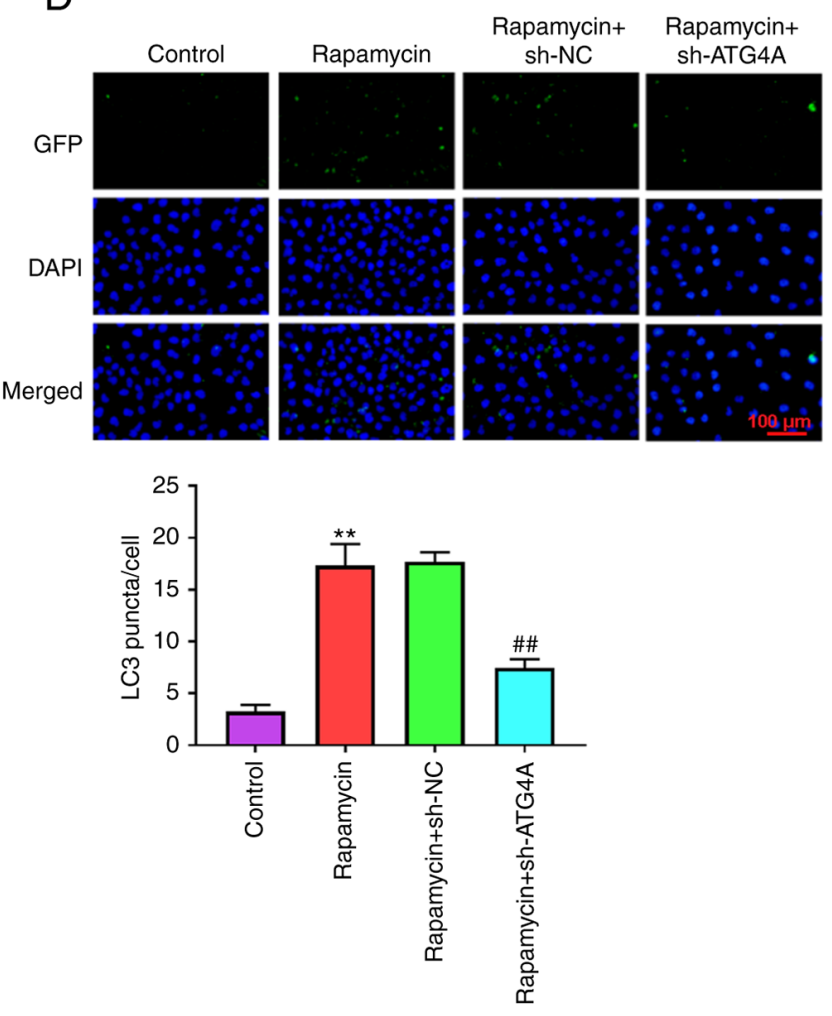

Figure 4. ATG4A knockdown attenuates tamoxifen-induced autophagy in MCF7/R cells. (A) After treatment with 4-OHT, the expression of LC3 and p62 in MCF7/R cells was determined using western blot analysis. (B) After treatment with rapamycin, the expression of LC3 and p62 in MCF7/R cells was determined using western blot analysis. (C) After treatment with 4-OHT, the number of LC3-puncta in MCF7/R cells was counted (scale bar, $100 \mu \mathrm{m}$ ). (D) After treatment with rapamycin, the number of LC3-puncta in MCF7/R cells was counted (scale bar, $100 \mu \mathrm{m}$ ). ${ }^{* *} \mathrm{P}<0.01$ vs. control group; ${ }^{\# \#} \mathrm{P}<0.01$ vs. $4-\mathrm{OHT}+$ sh-NC or rapamycin + sh-NC group. ATG4A, autophagy-related 4A; 4-OHT, 4-hydroxytamoxifen; sh-, short hairpin; NC, negative control.

revealed that patients with breast cancer are more responsive to endocrine therapy agents, such as selective ER modulators (SERMs), aromatase inhibitors and selective ER downregulators $(27,28)$. Tamoxifen, which is one of the SERMs, is a commonly used drug for treating ER positive breast cancer (4). However, the efficacy of tamoxifen is limited due to primary or acquired resistance $(5,29-31)$. Thus, it is important to increase the sensitivity of breast cancer to tamoxifen. The present 
A
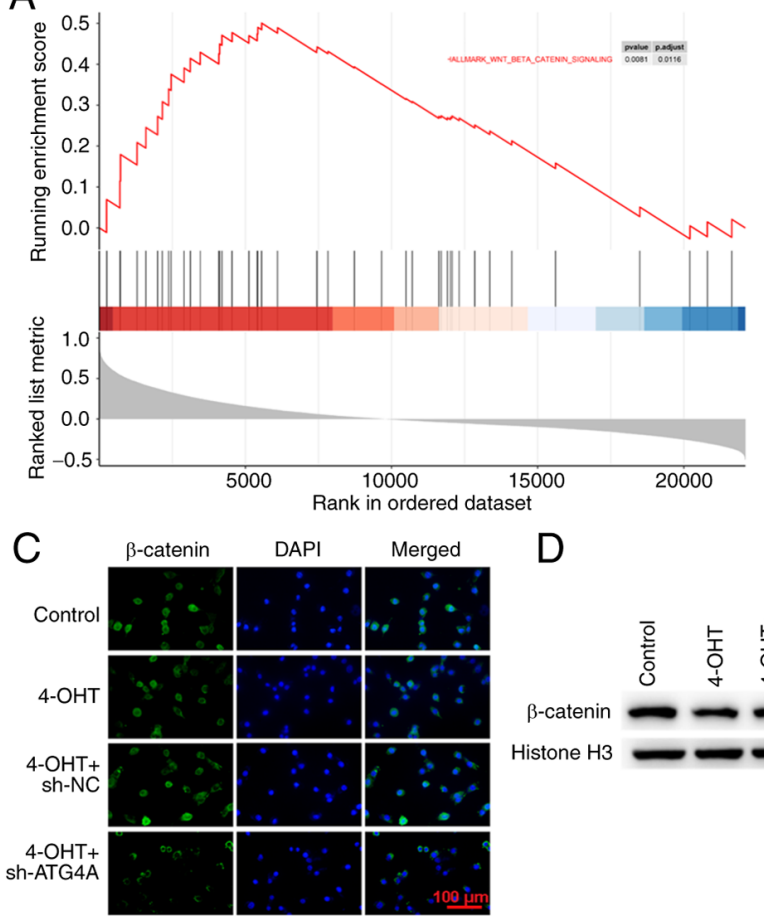

F

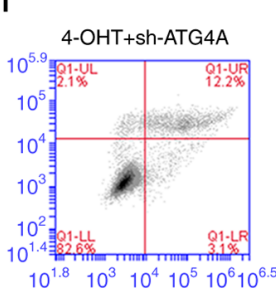

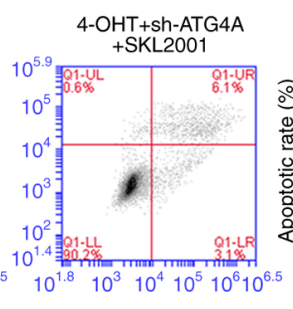

D
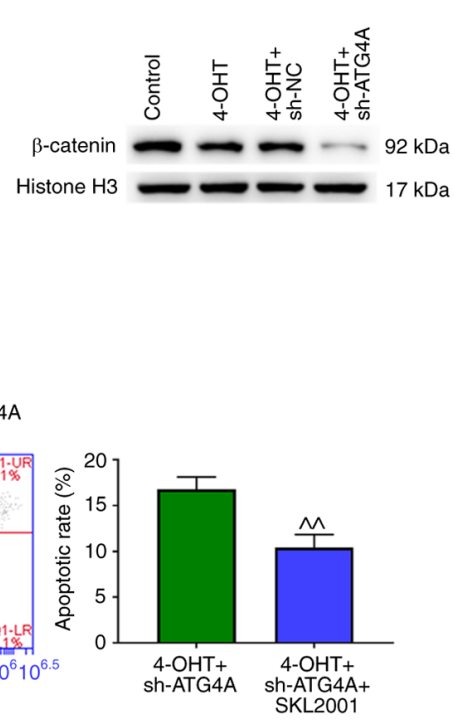

B
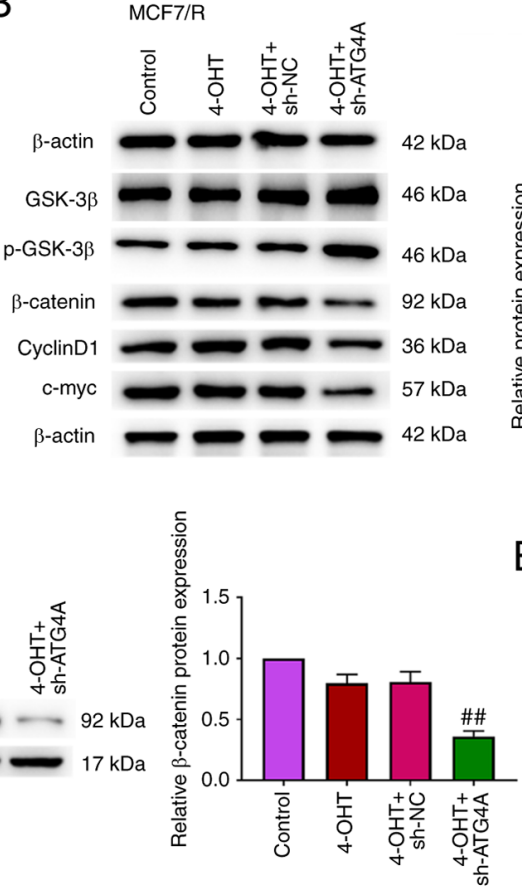

G

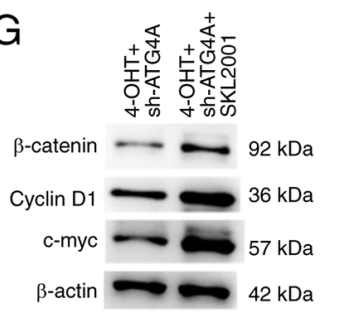

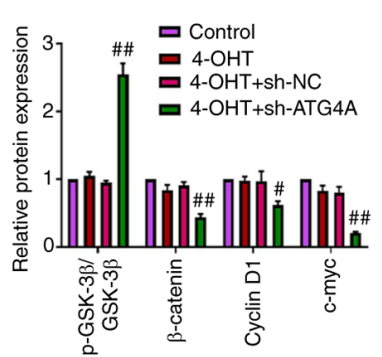

E $\rightarrow 4-O H T+$ sh-ATG4A E $\quad-4-\mathrm{OHT}+$ Sh-ATG4A+SKL2001
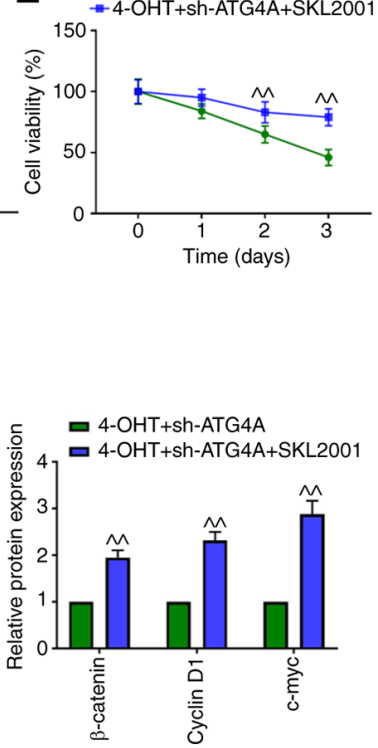

Figure 5. ATG4A knockdown dysregulates the Wnt/ $\beta$-catenin pathway in MCF7/R cells. (A) Gene set enrichment analysis indicated the association between ATG4A and Wnt/ $\beta$-catenin pathway. (B) The protein expression of p-GSK-3 $\beta$, GSK-3 $\beta$, $\beta$-catenin, cyclin D1 and c-myc was examined using western blot analysis. (C) ATG4A knockdown promoted the translocation of $\beta$-catenin from the nucleus to the cytoplasm in MCF7/R cells (scale bar, $100 \mu \mathrm{m}$ ). (D) ATG4A knockdown decreased $\beta$-catenin protein level in MCF7/R cells. (E) Cell viability was detected using Cell Counting Kit-8 assay. (F) Apoptosis was detected using flow cytometric analysis. (G) Western blot analysis was performed to detect the protein expression of $\beta$-catenin, cyclin D1 and c-myc. ${ }^{\wedge \wedge} \mathrm{P}<0.01$ vs. $4-\mathrm{OHT}+$ sh-ATG4A group; ${ }^{\#} \mathrm{P}<0.05$ and ${ }^{\# \#} \mathrm{P}<0.01$ vs. 4-OHT + sh-NC group. ATG4A, autophagy-related 4A; 4-OHT, 4-hydroxytamoxifen; sh-, short hairpin; NC, negative control; p, phosphorylated.

study suggested that downregulation of ATG4A inhibits the proliferation of breast cancer cells and overcomes tamoxifen resistance.

ATG4A, which is an autophagy-regulating molecule, is overexpressed during malignant progression of cancer (32). For instance, ATG4A expression is increased in osteosarcoma tissues, and its overexpression enhances the proliferation of osteosarcoma cell lines (14). Yang et al (15) identified that the expression of ATG4A is upregulated in gastric cancer tissues and its overexpression promotes cell migration and invasion. The present study revealed that a higher expression of ATG4A was associated with a poor prognosis in breast cancer. In addition, an increase in ATG4A was observed in tamoxifen-resistant cells compared with the control group. Autophagy is a protective housekeeping mechanism that maintains cellular metabolism under nutrient-limiting conditions, which is implicated in tumor development (33). LC3 is commonly used as an indicator for detecting autophagy (34). During the formation of autophagosomes, LC3 is cleaved by
ATG4 to form LC3-I, then cleaved-LC3 is conjugated with phosphatidylethanolamine to regulate the conversion of LC3-I to LC3-II. (35) Several studies suggested that ATG4 family members promote tumor progression. For example, the knockdown of ATG4B suppresses the survival of chronic myeloid leukemia cells, and promotes imatinib mesylate chemosensitivity by suppressing autophagy (36). The knockdown of ATG4C impairs autophagy, inhibits glioma progression, and sensitizes these cells to temozolomide (37). Studies have reported that 4-OHT stimulates autophagy in breast cancer, and inhibition of autophagy enhances the sensitivity of breast cancer cells to $4-\mathrm{OHT}(2,38,39)$. In the present study, treatment with 4-OHT significantly increased the LC3-II/LC3-I ratio and decreased the level of p62 (a marker of autophagy), while the knockdown of ATG4A enhanced the sensitivity of breast cancer cells to 4-OHT by inhibiting autophagy. Consistent with the findings of the present study, another study reported that overexpression of microRNA-24-3p led to reduction in ATG4A expression, allowing etoposide/cisplatin-resistant 
small-cell lung cancer cells to become sensitive to etoposide and cisplatin (21).

The Wnt/ $\beta$-catenin pathway is one of the most crucial pathways in regulating proliferation, cell cycle and death (40). When the Wnt/ $\beta$-catenin pathway is inactivated, nuclear $\beta$-catenin is transferred into the cytoplasm (41). Inhibition of GSK-3 $\beta$ phosphorylation promotes $\beta$-catenin degradation and changes the expression of cyclin D1 and c-myc (42). The results of the present study confirmed that ATG4A knockdown inhibited the Wnt/ $\beta$-catenin pathway in MCF7/R cells treated with 4-OHT. Consistent with the present research, overexpression of protein crumbs homolog 3 enhances tamoxifen sensitivity of tamoxifen-resistant cells by inhibiting $\beta$-catenin signaling (43). MS-275 (a histone deacetylase inhibitor) combined with cisplatin has been indicated to inhibit cell proliferation and migration and increase cell apoptosis by regulating the $\mathrm{Wnt} / \beta$-catenin pathway in esophageal squamous cell carcinoma cells (44). In addition, SKL2001, an agonist of the Wnt/ $\beta$-catenin pathway, effectively rescued the ATG4A knockdown-mediated anti-proliferative effect of tamoxifen-resistant cells. Consistent with the current study, a previous study demonstrated that SKL2001 rescued the WSB2-WD repeat and SOCS box containing 2 knockdown-mediated inhibition of cell proliferation in melanoma (45).

In summary, it was demonstrated that knockdown of ATG4A in tamoxifen-resistant cells reduced autophagy and suppressed the Wnt/ $\beta$-catenin pathway. ATG4A may affect the response to tamoxifen therapy of patients with breast cancer.

\section{Acknowledgements}

Not applicable.

\section{Funding}

No funding was received.

\section{Availability of data and materials}

The datasets used and/or analyzed during the current study are available from the corresponding author on reasonable request.

\section{Authors' contributions}

QL designed the experiments. LZ performed the research. QL analyzed the data and wrote the manuscript. QL and LZ confirmed the authenticity of all the raw data. Both authors read and approved the final version of the manuscript.

\section{Ethics approval and consent to participate}

Not applicable.

\section{Patient consent for publication}

Not applicable.

\section{Competing interests}

The authors declare that they have no competing interests.

\section{References}

1. Bray F, Ferlay J, Soerjomataram I, Siegel RL, Torre LA and Jemal A: Global cancer statistics 2018: GLOBOCAN estimates of incidence and mortality worldwide for 36 cancers in 185 countries. CA Cancer J Clin 68: 394-424, 2018.

2. Schoenlein PV, Periyasamy-Thandavan S, Samaddar JS, Jackson WH and Barrett JT: Autophagy facilitates the progression of ERalpha-positive breast cancer cells to antiestrogen resistance. Autophagy 5: 400-403, 2009.

3. Jennings CJ, Zainal N, Dahlan IM, Kay EW, Harvey BJ and Thomas W: Tamoxifen suppresses the growth of malignant pleural mesothelioma cells. Anticancer Res 36: 5905-5913, 2016.

4. Shagufta and Ahmad I: Tamoxifen a pioneering drug: An update on the therapeutic potential of tamoxifen derivatives. Eur J Med Chem 143: 515-531, 2018.

5. Early Breast Cancer Trialists' Collaborative Group (EBCTCG); Davies C, Godwin J, Gray R, Clarke M, Cutter D, Darby S, McGale P, Pan HC, Taylor C, et al: Relevance of breast cancer hormone receptors and other factors to the efficacy of adjuvant tamoxifen: Patient-level meta-analysis of randomised trials. Lancet 378: 771-784, 2011.

6. Nass $\mathrm{N}$ and Kalinski T: Tamoxifen resistance: From cell culture experiments towards novel biomarkers. Pathol Res Pract 211: 189-197, 2015.

7. Li YJ, Lei YH, Yao N, Wang CR, Hu N, Ye WC, Zhang DM and Chen ZS: Autophagy and multidrug resistance in cancer. Chin J Cancer 36: 52, 2017.

8. Liu J, Yue W and Chen H: The correlation between autophagy and tamoxifen resistance in breast cancer. Int J Clin Exp Pathol 12: 2066-2074, 2019.

9. Samaddar JS, Gaddy VT, Duplantier J, Thandavan SP, Shah M, Smith MJ, Browning D, Rawson J, Smith SB, Barrett JT and Schoenlein PV: A role for macroautophagy in protection against 4-hydroxytamoxifen-induced cell death and the development of antiestrogen resistance. Mol Cancer Ther 7: 2977-2987, 2008

10. Klionsky DJ: Citing recent declines in the discovery of new ATG genes, some scientists now suggest that the end of autophagy research may be within sight. Autophagy 10: 715-716, 2014.

11. Zhang L, Li J, Ouyang L, Liu B and Cheng Y: Unraveling the roles of Atg 4 proteases from autophagy modulation to targeted cancer therapy. Cancer Lett 373: 19-26, 2016.

12. Mariño G, Uría JA, Puente XS, Quesada V, Bordallo J and López-Otín C: Human autophagins, a family of cysteine proteinases potentially implicated in cell degradation by autophagy. J Biol Chem 278: 3671-3678, 2003.

13. Betin VM and Lane JD: Atg4D at the interface between autophagy and apoptosis. Autophagy 5: 1057-1059, 2009.

14. Su H, Zhu G, Rong X,Zhou Y, Jiang P and Chen P: Upregulation of ATG4A promotes osteosarcoma cell epithelial-to-mesenchymal transition through the Notch signaling pathway. Int J Clin Exp Pathol 10: 7975-7982, 2017.

15. Yang SW, Ping YF, Jiang YX, Luo X, Zhang X, Bian XW and Yu PW: ATG4A promotes tumor metastasis by inducing the epithelial-mesenchymal transition and stem-like properties in gastric cells. Oncotarget 7: 39279-39292, 2016.

16. Wolf J, Dewi DL, Fredebohm J, Müller-Decker K, Flechtenmacher C, Hoheisel JD and Boettcher M: A mammosphere formation RNAi screen reveals that ATG4A promotes a breast cancer stem-like phenotype. Breast Cancer Res 15: R109, 2013.

17. Colaprico A, Silva TC, Olsen C, Garofano L, Cava C, Garolini D, Sabedot TS, Malta TM, Pagnotta SM, Castiglioni I, et al: TCGAbiolinks: An R/Bioconductor package for integrative analysis of TCGA data. Nucleic Acids Res 44: e71, 2016.

18. Therneau TM: Survival Analysis [R package survival version 2.41-3]. Technometrics 46: 111-112, 2015.

19. Yan C, Zhao J, Qin Y, Zhao F, Ji L and Zhang J: Overexpression of ATG4a promotes autophagy and proliferation, and inhibits apoptosis in lens epithelial cells via the AMPK and Akt pathways. Mol Med Rep 22: 1295-1302, 2020.

20. Guo L, Zhou L, Gao Q, Zhang A, Wei J, Hong D, Chu Y, Duan X, Zhang Y and Xu G: MicroRNA-144-3p inhibits autophagy activation and enhances Bacillus Calmette-Guérin infection by targeting ATG4a in RAW264.7 macrophage cells. PLoS One 12: e0179772, 2017.

21. Pan B, Chen Y, Song H, Xu Y, Wang R and Chen L: Mir-24-3p downregulation contributes to VP16-DDP resistance in small-cell lung cancer by targeting ATG4A. Oncotarget 6: 317-331, 2015. 
22. Uppada SB, Gowrikumar S, Ahmad R, Kumar B, Szeglin B, Chen X, Smith JJ, Batra SK, Singh AB and Dhawan P: MASTL induces colon cancer progression and chemoresistance by promoting Wnt $/ \beta$-catenin signaling. Mol Cancer 17 : $111,2018$.

23. Ohashi W, Yamamine N, Imura J and Hattori Y: SKL2001 suppresses colon cancer spheroid growth through regulation of the E-cadherin/ $\beta$-Catenin complex. Biochem Biophys Res Commun 493: 1342-1348, 2017.

24. Siegel RL, Miller KD and Jemal A: Cancer statistics, 2016. CA Cancer J Clin 66: 7-30, 2016.

25. Gianni L, Baselga J, Eiermann W, Porta VG, Semiglazov V, Lluch A, Zambetti M, Sabadell D, Raab G, Cussac AL, et al: Phase III trial evaluating the addition of paclitaxel to doxorubicin followed by cyclophosphamide, methotrexate, and fluorouracil, as adjuvant or primary systemic therapy: European cooperative trial in operable breast cancer. J Clin Oncol 27: 2474-2481, 2009.

26. Erol K, Baltali E, Altundag K, Guler N, Ozisik Y, Onat DA, Sayek I, Cengiz M, Atahan L and Tekuzman G: Neoadjuvant chemotherapy with cyclophosphamide, mitoxantrone, and 5-fluorouracil in locally advanced breast cancer. Onkologie 28: 81-85, 2005.

27. Harbeck N and Gnant M: Breast cancer. Lancet 389: 1134-1150, 2017.

28. Ignatiadis $M$ and Sotiriou C: Luminal breast cancer: From biology to treatment. Nat Rev Clin Oncol 10: 494-506, 2013.

29. Mansouri S, Feizi N, Mahdi A, Majidzadeh-AK and Farahmand L: A review on the role of VEGF in tamoxifen resistance. Anticancer Agents Med Chem 18: 2006-2009, 2018.

30. Szostakowska M, Trębińska-Stryjewska A, Grzybowska EA and Fabisiewicz A: Resistance to endocrine therapy in breast cancer: Molecular mechanisms and future goals. Breast Cancer Res Treat 173: 489-497, 2019.

31. Ali S, Mondal N, Choudhry H, Rasool M, Pushparaj PN, Khan MA, Mahfooz M, Sami GA, Jarullah J, Ali A and Jamal MS: Current management strategies in breast cancer by targeting key altered molecular players. Front Oncol 6: 45, 2016.

32. Du JX, Chen C, Luo YH, Cai JL, Cai CZ, Xu J, Ni XJ and Zhu W: Establishment and validation of a novel autophagy-related gene signature for patients with breast cancer. Gene 762: 144974 2020.

33. Bhat P, Kriel J, Shubha Priya B, Basappa, Shivananju NS and Loos B: Modulating autophagy in cancer therapy: Advancements and challenges for cancer cell death sensitization. Biochem Pharmacol 147: 170-182, 2018.

34. Mizushima N: Methods for monitoring autophagy using GFP-LC3 transgenic mice. Methods Enzymol 452: 13-23, 2009.

35. Ravikumar B, Sarkar S, Davies JE, Futter M, Garcia-Arencibia M, Green-Thompson ZW, Jimenez-Sanchez M, Korolchuk VI, Lichtenberg M, Luo S, et al: Regulation of mammalian autophagy in physiology and pathophysiology. Physiol Rev 90: 1383-1435, 2010.
36. Rothe K, Lin H, Lin KB, Leung A, Wang HM, Malekesmaeili M, Brinkman RR, Forrest DL, Gorski SM and Jiang X: The core autophagy protein ATG4B is a potential biomarker and therapeutic target in CML stem/progenitor cells. Blood 123: 3622-3634, 2014

37. Wen ZP, Zeng WJ, Chen YH, Li H, Wang JY, Cheng Q, Yu J, Zhou HH, Liu ZZ, Xiao J and Chen XP: Knockdown ATG4C inhibits gliomas progression and promotes temozolomide chemosensitivity by suppressing autophagic flux. J Exp Clin Cancer Res 38: 298, 2019.

38. PawlikA,Słomińska-WojewódzkaMandHerman-Antosiewicz A: Sensitization of estrogen receptor-positive breast cancer cell lines to 4-hydroxytamoxifen by isothiocyanates present in cruciferous plants. Eur J Nutr 55: 1165-1180, 2016.

39. Yu X, Luo A, Liu Y, Wang S, Li Y, Shi W, Liu Z and Qu X: MiR-214 increases the sensitivity of breast cancer cells to tamoxifen and fulvestrant through inhibition of autophagy. Mol Cancer 14: 208, 2015.

40. Yu J, Liu D, Sun X, Yang K, Yao J, Cheng C, Wang C and Zheng J: CDX2 inhibits the proliferation and tumor formation of colon cancer cells by suppressing Wnt $/ \beta$-catenin signaling via transactivation of GSK-3 $\beta$ and Axin2 expression. Cell Death Dis 10: 26, 2019.

41. Xu C, Liu F, Xiang G, Wang S, Liu J, Meng Q, Xu D, Lv S, Jiao J and Niu Y: $\beta$-Catenin nuclear localization positively feeds back on EGF/EGFR-attenuated AJAP1 expression in breast cancer. J Exp Clin Cancer Res 38: 238, 2019.

42. Liu S, Wang L, Ding W, Wang D, Wang X, Luo Q, Lu Y and Zhu L: Cleistanthin A inhibits the invasion of MDA-MB-231 human breast cancer cells: Involvement of the $\beta$-catenin pathway. Pharmacol Rep 72: 188-198, 2020.

43. Li P, Feng C, Chen H, Jiang Y, Cao F, Liu J and Liu P: Elevated CRB3 expression suppresses breast cancer stemness by inhibiting $\beta$-catenin signalling to restore tamoxifen sensitivity. J Cell Mol Med 22: 3423-3433, 2018

44. Liu T, Wang Y, Zhang Z, Zhang Z, Li Y, Cui Y, Li Z, Liu H, Zhang Y, Wang Y and Ma S: MS-275 combined with cisplatin exerts synergistic antitumor effects in human esophageal squamous cell carcinoma cells. Toxicol Appl Pharmacol 23: 114971, 2020.

45. Zhang Y, Li Z, Zhao W, Hu H, Zhao L, Zhu Y, Yang X, Gao B, Yang $\mathrm{H}$, Huang $\mathrm{Y}$ and Song $\mathrm{X}$ : WD repeat and SOCS box containing protein 2 in the proliferation, cycle progression, and migration of melanoma cells. Biomed Pharmacother 116: 108974, 2019.

(7) $\Theta$ This work is licensed under a Creative Commons Attribution-NonCommercial-NoDerivatives 4.0 International (CC BY-NC-ND 4.0) License. 